\title{
LATTICES OF TOPOLOGICAL EXTENSIONS
}

\author{
BY \\ JOHN MACK, MARLON RAYBURN AND GRANT WOODS
}

\begin{abstract}
For completely regular Hausdorff spaces, we consider topological properties $\boldsymbol{P}$ which are akin to compactness in the sense of Herrlich and van der Slot and satisfy the equivalent of Mrowka's condition (W). The algebraic structure of the family of tight extensions of $X$ (which have $P$ and contain no proper extension with that property) is studied. Where $X$ has $P$ locally but not globally, the relations between the complete lattice $P^{*}(X)$ of those tight extensions which are above the maximal one-point extension and the topology of the $P$-reflection are investigated and conditions found under which $P^{*}(X)$ characterizes $\gamma X-X$. The results include those of Magill on the lattice of compactifications of a locally compact space, and other applications are considered.
\end{abstract}

1. Introduction. All spaces considered in this paper are assumed to be completely regular and Hausdorff.

Attaching an algebraic object to a topological object in order to study the latter is an old and honorable procedure. Two excellent examples of this are afforded by the use [5] of the ring of continuous functions to study a space $X$, and the use of the semilattice of compactifications to study the outgrowth of its Stone-Cech compactification, $\beta X-X$. In [9], K.D. Magill, Jr. has established the result which we shall hereafter refer to as

Magill's Theorem. If $X$ and $Y$ are locally compact Hausdorff spaces, then $\beta X-X$ is homeomorphic to $\beta Y-Y$ if and only if the lattices of compactifications $K(X)$ and $K(Y)$ are lattice isomorphic.

Motivated by this and our previous work [8] on local topological properties, we have found that compactness is only one of a class of topological properties for which there is a relationship [\$4] between a semilattice of (tight) extensions [\$3] and the topology of the outgrowth of a certain extension (the $P$-reflection) [\$2]. This class also includes such things as realcompactness, almost realcompactness and $m$-boundedness. These examples will be discussed in $\$ 5$ below.

2. The property $P$ and locally- $P$ spaces. Topological properties in the class in which we are interested may be described by

2.1. Definition. Let $P$ be any topological property that is (a) closed-hereditary, (b) productive under arbitrary products and (c) such that if $X$ is the union of a compact space and a space with $P$, then $X$ has $P$.

Presented to the Society, August 10, 1971 under the title Lattices of topological extensions for locally P-spaces; received by the editors September 22, 1971 and, in revised form, June 20, 1972.

AMS (MOS) subject classifications (1970). Primary 54D40, 54D60; Secondary 54D35, 06A23.

Key words and phrases. $P$-reflection, lattice of tight $P$-extensions, compact, realcompact, almost realcompact, $m$-bounded.

Copyright $\Theta 1974$, American Mathematical Society 
Since we allow the empty set to possess property $P$ vacuously, (c) insures that every compact space has $P$. Properties satisfying (a) and (b) are studied in [7], and from there we obtain

2.2. Proposition. If $C$ is an arbitrary collection of subspaces of some fixed space and if every member of $C$ has $P$, then $\cap C$ has $P$.

2.3. Definition. For any space $X, \gamma X=\cap\{T: T$ has $P$ and $X \subseteq T \subseteq \beta X\}$ will be called the "P-reflection" of $X$. If $P$ is compactness, $\gamma X=\beta X$; if $P$ is realcompactness, $\gamma X=v X$ (the Hewitt realcompactification).

2.4. Corollary. The P-reflection of any space has $P$.

2.5. Theorem. Property $P$ satisfies Mrowka's condition (W): if a point $x_{0}$ of a space $X$ has a neighborhood base $B$ such that, for each $B$ in $B, X-B$ has $P$, then $X$ has $P$.

Proof. Let $B$ be in $B$. By 2.1(c), $X \cup \mathrm{cl}_{\beta X} B=(X-B) \cup \mathrm{cl}_{\beta X} B$ has $P$. Moreover if $y \in \mathrm{cl}_{\beta X} B-X$, then there is a $\beta X$-open neighborhood $G$ of $x_{0}$ whose closure in $\beta X$ misses $y$. Let $T$ be a member of $B$ which is contained in $G$. Then $y \notin X \cup \operatorname{cl}_{\beta X} T$. Hence $X=\cap\left\{X \cup \operatorname{cl}_{\beta X} B: B \in \mathcal{B}\right\}$, which by 2.2 has $P$.

For a further discussion of Mrowka's condition (W), see [12].

2.6. Corollary. For any closed-hereditary productive property, Mrowka's condition (W) is equivalent to 2.1(c).

Proof. Theorem 2.3(c) of [8].

2.7. Corollary. If $X$ has $P$ and if $Y$ is the (completely regular Hausdorff) quotient space obtained by identifying the points of a compact subset of $X$, then $Y$ has $P$.

Proof. Theorem 2.3(a) of [8].

2.8. Definition. Let $X$ be any space and let $x \in X$. We say $X$ is locally-P at $x$ if there is an $X$-neighborhood of $x$ with $P$. $X$ is locally-P if it is locally-P at every point, and 1.p. if it is locally-P but does not have $P$. Let $W(X)=\{x \in X: X$ is not locally-P at $x$.

It is shown in [8] that if $X$ does not have $P$, then there is a one-point extension of $X$ with $P$ if and only if $X$ is locally-P. We call the largest such extension ${ }^{*} X$. If ${ }^{*} X-X=\{\omega\}$, then ${ }^{*} X$ has the quotient topology induced by that mapping from $X \cup \operatorname{cl}_{\beta X}(\gamma X-X)$ onto ${ }^{*} X$ which fixes $X$ pointwise and maps each point of $\operatorname{cl}_{\beta X}(\gamma X-X)$ to $\omega$. See Theorem 4.1 of [8] for further details.

2.9. Definition. Let $X$ not have $P$. Set $K_{X}=\operatorname{cl}_{\beta X}(\gamma X-X)$ and $\delta X=X$ $\cup\left(\beta X-K_{X}\right)$.

2.10. Lemma. $W(X)=X \cap K_{X}$.

Proof. Let $x \in X-K_{X}$. Then $\gamma X-K_{X}$ is a $\gamma X$-open neighborhood of $x$. As $\gamma X$ is regular, there is a $\gamma X$-closed set $F$ with $x \in$ int $_{\gamma} F \subseteq F \subseteq \gamma X-K_{X} \subseteq X$. By 2.1(a), $F$ has $P$. Thus $x \notin W(X)$, so $W(X) \subseteq X \cap K_{X}$. 
Conversely, let $x \in$ int $_{X} G$ where $G$ has $P$. Let $H=\operatorname{cl}_{\beta X}\left(X-\right.$ int $\left._{X} G\right)$, so $H$ is compact and $X \subseteq G \cup H$, which has $P$ by 2.7. Since $G \cup H \subseteq \beta X$, we have $\gamma X \subseteq G \cup H$ and $\gamma X=\gamma X \cap(G \cup H)=G \cup(\gamma X \cap H)=G \cup \bar{G}$, where $\bar{G}=\operatorname{cl}_{\gamma X}\left(X-\right.$ int $\left._{X} G\right)$. Since $\bar{G} \cap X=X-$ int $_{X} G$, we have $\gamma X-\bar{G}$ $=$ int $_{X} G$. Thus int ${ }_{X} G$ is open in $\gamma X, x \in$ int $_{X} G$ and (int $\left.{ }_{X} G\right) \cap(\gamma X-X)=\varnothing$. Whence $x \notin K_{X}$ and $W(X)=K_{X} \cap X$.

2.11. Corollary. The following are equivalent:

(a) $X$ is locally-P.

(b) $\delta X$ is locally compact.

(c) $X$ is open in $\gamma X$.

3. The partially ordered set of tight $\boldsymbol{P}$-extensions.

3.1. Definition. (a) Let $X$ be contained densely in a space $T$ that has $P$. If there exists no space $V$ such that $X \subseteq V \subseteq T$ and $V$ has $P$, then $T$ will be called a tight $P$-extension of $X$. Two tight $P$-extensions of $X$ are equivalent if there is a homeomorphism from one onto the other that fixes $X$ pointwise. Let $P(X)$ be the resulting family of equivalence classes of tight $P$-extensions.

(b) Let $X$ be a fixed space and let $T_{1}$ and $T_{2}$ be in $P(X)$. Set $T_{1} \leq T_{2}$ if there is a continuous mapping from $T_{2}$ into $T_{1}$ which fixes $X$ pointwise. It follows immediately, see $0.12(a)$ of [5], that $(P(X), \leq)$ is a partially ordered set.

Notice that if the property concerned is compactness, every compactification is automatically tight and $P(X)=K(X)$ is the usual family of compactifications. This case has been well studied [1], [9]; it is known that $K(X)$ is a complete upper semilattice which is conditionally complete (every family of compactifications with a lower bound has an infimum) and $K(X)$ is a complete lattice if and only if $X$ is locally compact.

3.2. Lemma. Let $T_{1}$ and $T_{2}$ be in $P(X)$. Then $T_{1} \leq T_{2}$ in $P(X)$ if and only if $\beta T_{1} \leq \beta T_{2}$ in $K(X)$.

Proof. Suppose $\beta T_{1} \leq \beta T_{2}$. Then there is a continuous surjection $f: \beta T_{2} \rightarrow \beta T_{1}$ that leaves $X$ pointwise fixed. If $f\left[T_{2}\right]-T_{1} \neq \varnothing$, then $T_{2}-f^{\leftarrow}\left[T_{1}\right] \neq \varnothing$. From Proposition 1 of [7], $f^{\leftarrow}\left[T_{1}\right]$ has $P$. Thus by $2.2, f^{\leftarrow}\left[T_{1}\right] \cap T_{2}$ has $P$ and is a proper subset of $T_{2}$ that contains $X$. This contradicts the tightness of $T_{2}$. Hence $f\left[T_{2}\right] \subseteq T_{1}$, so the restriction of $f$ to $T_{2}$ takes $T_{2}$ into $T_{1}$ and leaves $X$ pointwise fixed, i.e. $T_{1} \leq T_{2}$.

Conversely, if $T_{1} \leq T_{2}$, let $g$ map $T_{2}$ into $T_{1}$ and fix $X$ pointwise. Then $g$ extends to a continuous map $g^{\beta}: \beta T_{2} \rightarrow \beta T_{1}$ that fixes $X$ pointwise (6.5 of [5]). Hence $\beta T_{1} \leq \beta T_{2}$.

3.3. Theorem. $P(X)$ is a complete upper semilattice which is conditionally complete. Moreover, it is a complete lattice if and only if $X$ is locally compact or has $P$.

Proof. Let $\varnothing \neq \Im \subseteq P(X)$ and let $\alpha X$ be the supremum in $K(X)$ of $\{\beta T: T \in \Im\}$. Let $T_{0}=\cap\{V: X \subseteq V \subseteq \alpha X$ and $V$ has $P\}$. Then $T_{0} \in P(X)$ 
and since $\alpha X$ is a compactification of $T_{0}$, we have $\beta T \leq \alpha X \leq \beta T_{0}$ for every $T \in \mathcal{Y}$. Suppose there is some $T_{2}$ in $P(X)$ such that $\beta T \leq \beta T_{2}$ for every $T \in \mathcal{Y}$. Then $\alpha X \leq \beta T_{2}$. A repetition of the first part of the proof of 3.2 (with $\alpha X$ in place of $\beta T_{1}$ ) shows that $T_{0} \leq T_{2}$. Thus $T_{0}$ is the supremum of $\rightarrow$ in $P(X)$. In a similar fashion we use the lower conditional completeness of $K(X)$ to show the lower conditional completeness of $P(X)$. Again, application of 3.2 shows that $\gamma X$ is the supremum of $P(X)$ and that $P(X)$ has an infimum if and only if the Alexandrov compactification $X^{*}$ exists.

Recollect that a continuous surjection is called a perfect map if it is closed and the preimage of any point is compact. As remarked before, if $X$ is l.p., then there is a largest one-point extension ${ }^{*} X$ in $P(X)$.

3.4. Lemma. Let $T \in P(X)$ and let $f$ map $\beta X$ onto $\beta T$ and fix $X$ pointwise. The following are equivalent:

(a) The restriction of $f$ to $X \cup K_{X}$ is a perfect map.

(b) The restriction of $f$ to $X \cup K_{X}$ is a quotient map.

(c) The restriction of $f$ to $\delta X$ is a homeomorphism.

Moreover if $X$ is a proper open subset of $\gamma X$, these are equivalent to

(d) $* X \leq T$.

Proof. (b) implies (c). It suffices to show that if $t \in \beta X-\left(X \cup K_{X}\right)$ and $s \in \beta X$, then $t \neq s$ implies $f(t) \neq f(s)$. If $t \neq s$, there exists a continuous, realvalued $h$ on $\beta X$ such that $0 \leq h \leq 1$, while $h(t)=0$ and $h\left[K_{X} \cup\{s\}\right]=\{1\}$. Set $g=f \mid X \cup K_{X}$ and define $k: f\left[X \cup K_{X}\right] \rightarrow[0,1]$ so that $k \circ g=h \mid X \cup K_{X}$. It follows from the construction of $h$ that $k$ is well defined. Since $g$ is a quotient map by assumption, $k$ is continuous. Let $k^{\beta} \in C(\beta T)$ be the extension of $k$ (note that there is no ambiguity here since $T \subseteq f\left[X \cup K_{X}\right] \subseteq \beta T$ implies $\left.\beta\left(f\left[X \cup K_{X}\right]\right)=\beta T\right)$. Since $k^{\beta} \circ f$ agrees with $h$ on the dense subspace $X$ of $\beta T$, we have $h=k^{\beta} \circ f$. But $h(t) \neq h(s)$, so $f(t) \neq f(s)$.

(c) implies (a). By Theorem 6.11 of [5], $f\left[K_{X}-X\right]=\beta T-f[\delta X]$, so $K_{X}-X$ $=f^{\leftarrow} \circ f\left[K_{X}-X\right]$. Also $X=f^{\leftarrow} \circ f[X]$. Thus $X \cup K_{X}=f^{\leftarrow} \circ f\left[X \cup K_{X}\right]$. This is sufficient to get (a).

(b) implies (d). Let $X$ be open in $\gamma X$ and let ${ }^{*} X-X=\{\omega\}$. Define $g: T \rightarrow{ }^{*} X$ by $g(x)=x$ if $x \in X$ and $g(t)=\omega$ if $t \in T-X$. If $g$ is continuous, then ${ }^{*} X \leq T$. Note that if $h=f \mid X \cup K_{X}$, then $g \circ h$ leaves $X$ pointwise fixed and maps all points of $K_{X}$ to $\omega$. This map is continuous by definition of ${ }^{*} X$. Since $T$ has the quotient topology, $g$ is continuous.

(d) implies (c). Let $g: T \rightarrow{ }^{*} X$ be continuous and leave $X$ pointwise fixed. Let $h: X \cup K_{X} \rightarrow{ }^{*} X$ be the continuous map that leaves $X$ pointwise fixed and maps $K_{X}$ to $\{\omega\}={ }^{*} X-X$. Let $g^{\beta}: \beta T \rightarrow \beta\left({ }^{*} X\right)$ and $h^{\beta}: \beta X \rightarrow \beta\left({ }^{*} X\right)$ be the continuous extensions of $g$ and $h$. Then $h^{\beta}=g^{\beta} \circ f$. Since the equivalence of (a) and (c) applies to $h^{\beta}$, and since $h^{\beta} \mid X \cup K_{X}$ is evidently a perfect map, the restriction of $g^{\beta} \circ f$ to $\delta X$ is a homeomorphism. Hence the same is true of $f$. 
3.5. Definition. Let $X$ be an 1.p. space. Put $P^{*}(X)=\left\{T \in P(X):^{*} X \leq T\right\}$, and where $f$ is the map from $\beta X$ onto $\beta T$ that fixes $X$ pointwise, put $D^{*}(X)$ $=\left\{T \in P^{*}(X): f[\gamma X]=T\right\}$. Thus for a general 1.p. space, we have $D^{*}(X)$ $\subseteq P^{*}(X) \subseteq P(X)$. If $P$ is compactness, of course both are equalities. In the last section, we give an example of a locally realcompact space for which $D^{*}(X)$ $\neq P^{*}(X)$.

3.6. Proposition. If $X$ is l.p., then $P^{*}(X)$ is a complete lattice.

Proof. Follows immediately from 3.3.

Lemma 3.4 shows that the relations between decompositions of $\beta X-X$ and the compactifications of locally compact $X$, studied by Magill in [9], require only minor changes to describe the relations between decompositions of $K_{X}-X$ and extensions in $P^{*}(X)$ for any l.p. space. Since the more general theory requires essentially the same proofs, we state only a few of the lemmas to indicate the nature of the required changes and refer the reader to Lemmas 1-11 of Magill's paper.

3.7. Definition. Let $X$ be l.p. and let $T \in P^{*}(X)$. Let $f$ be the map from $\beta X$ onto $\beta T$ where $f$ fixes $X$ pointwise, and set $\mathcal{Y}(T)=\left\{f^{\leftarrow}(p): p \in f\left[K_{X}\right]\right\}$. Then $\mathcal{Y}(T)$ is an upper semicontinuous decomposition of $K_{X}$ into compact subsets.

3.8. Lemma. Let $T_{1}$ and $T_{2}$ be in $P^{*}(X)$. Then $T_{1} \leq T_{2}$ if and only if $\Im\left(T_{2}\right)$ refines $\Im\left(T_{1}\right)$.

3.9. Lemma. Let $T \in P^{*}(X)$ and let $f$ map $\beta X$ onto $\beta T$ and fix $X$ pointwise. Let $\left(K_{i}\right)_{i=1}^{n}$ be a finite family of pairwise disjoint compact subsets of $f\left[K_{X}\right]$ such that $K_{i} \cap T \neq \varnothing$ for each $i$. The quotient space obtained from $\cup_{i=1}^{n}\left(T \cup K_{i}\right)$ by collapsing each $K_{i}$ to a point is also in $P^{*}(X)$.

3.10. Lemma. Let $\left(K_{i}\right)_{i=1}^{n}$ be a finite, pairwise disjoint family of compact subsets of $K_{X}$ such that for each $i, \gamma X \cap K_{i} \neq \varnothing$. There is a unique $T$, call it $T\left(K_{i}: i=1, \ldots, n\right)$, in $P^{*}(X)$ such that $\exists(T)$ consists of precisely the sets $K_{i}$ and all singletons $\{p\}$ with $p \in K_{X}-\cup_{i=1}^{n} K_{i}$.

3.11. Lemma. An element $T$ of $P^{*}(X)$ is an anti-atom of $P^{*}(X)$ if and only if there is a $p \in \gamma X-X$ and $q \in K_{X}, p \neq q$, such that $T=T(p, q)$. Notice this says every anti-atom of $P^{*}(X)$ is in $D^{*}(X)$.

3.12. Lemma. Let $T \in P^{*}(X)$. Then $\Im(T)$ has exactly one set larger than a singleton and not disjoint from $\gamma X$ if and only if $T \neq \gamma X$ and there does not exist an anti-atom $T_{1} \in P^{*}(X)$ such that (1) $T_{1} \wedge T \neq T$ and (2) for every anti-atom $T_{2} \in P^{*}(X)$ such that (a) $T_{1} \neq T_{2}$ and (b) $T_{1} \wedge T=T_{2} \wedge T$, the only anti-atoms greater than $T_{1} \wedge T_{2}$ are $T_{1}$ and $T_{2}$.

4. The main results. By 2.11 , if $X$ is $1 . p$. then $\delta X$ is locally compact and hence its family of compactifications $\underline{K}(\delta X)$ is a complete lattice. It is well known that for any space $X$, there is a space $Y$ and a perfect map $f: X \rightarrow Y$ if and only if $X$ 
admits an upper semicontinuous decomposition into compact sets, i.e. a "perfect decomposition". Every compactification of $X$ is uniquely determined by a perfect decomposition of $\mathrm{cl}_{\beta X}(\beta X-X)$ which consists of singletons on $X \cap$ $\mathrm{cl}_{\beta X}(\beta X-X)$. Since the compactifications of $\delta X$ are determined similarly by perfect decompositions of $\mathrm{cl}_{\beta X}(\beta X-\delta X)$ which consist of singletons on $\delta X \cap \operatorname{cl}_{\beta X}(\beta X-\delta X)=W(X)$, we have a natural way of identifying $K(\delta X)$ and compactifications of these two spaces. In particular if $X$ is 1.p., we identify $\left\{\alpha X \in K(X): \beta\left(^{*} X\right) \leq \alpha X\right\}$.

4.1. Proposition. For any l.p. space, ${ }^{*}(\delta X)=(\delta X)^{*}=\beta\left({ }^{*} X\right)$.

Proof. Since $\delta X$ is locally compact, both ${ }^{*}(\delta X)$ and the Alexandrov compactification $(\delta X)^{*}$ exist. Now $X \subseteq \delta X \subseteq \gamma(\delta X) \subseteq \beta(\delta X)=\beta X$, hence $\gamma X$ $\subseteq \gamma(\delta X)$. So $\gamma X-\delta X=\gamma X-X \subseteq \gamma(\delta X)-\delta X \subseteq \beta(\delta X)-\delta X=K_{X}$ which is compact. Thus $K_{X}=\operatorname{cl}_{\beta X}(\gamma X-X) \subseteq \mathrm{cl}_{\beta(\delta X)}[\gamma(\delta X)-\delta X] \subseteq K_{X}$. Thus $\mathrm{cl}_{\beta(\delta X)}[\gamma(\delta X)-\delta X]=K_{X}=\beta(\delta X)-\delta X$. Now $*(\delta X)$ is constructed by collapsing $\operatorname{cl}_{\beta(\delta X)}[\gamma(\delta X)-\delta X]$ to a point and $(\delta X)^{*}$ is constructed by collapsing $\beta(\delta X)-\delta X$ to a point. Hence ${ }^{*}(\delta X)=(\delta X)^{*}$. Finally ${ }^{*} X$ is constructed by collapsing $K_{X}$ to a point. So $(\delta X)^{*}=\beta\left({ }^{*} X\right)$.

4.2. Corollary. If $X$ is l.p. and $\gamma X-X$ is compact, then $\gamma(\delta X)=\beta(\delta X)$, so $P(\delta X)=P^{*}(\delta X)=K(\delta X)$.

4.3. Proposition. If $X$ is l.p., then $P^{*}(X)$ is lattice-isomorphic to $K(\delta X)$ if and only if $\left|K_{X}-\gamma X\right| \leq 1$.

Proof. (If) Let $\tau: P^{*}(X) \rightarrow K(\delta X)$ be defined by $\tau(T)=\beta T$. Since we know $\tau$ is one-to-one, in light of 3.2, we need only show that $\tau$ is onto.

Let $E$ be in $K(\delta X)$ and consider $E$ as in $K(X)$ with $E \geq \beta\left({ }^{*} X\right)$. Then there is a $T$ in $P^{*}(X)$ with $T \subseteq E=\alpha T \leq \beta T$. Let $k: \beta T \rightarrow E$ be the map fixing $T$ pointwise. If $\alpha T \neq \beta T$, then for some $t$ in $\alpha T-T$, we have $\left|k^{\leftarrow}(t)\right|>1$. Thus there are (at least) two distinct points $x$ and $y$ in $\beta T-T$ such that $k(x)=k(y)$ $=t$. Let $f: \beta X \rightarrow \beta T$ fix $X$ pointwise and let $g: \beta X \rightarrow E$ fix $X$ pointwise. Let $x_{1}$ be in $f^{\leftarrow}(x)$ and $y_{1}$ be in $f^{\leftarrow}(y)$. Then $g\left(x_{1}\right)=g\left(y_{1}\right)=y$. But $g \mid \delta X$ is a homeomorphism, so by 6.11 of [5], neither $x_{1}$ nor $y_{1}$ is in $\delta X$. If either $x_{1}$ or $y_{1}$ is in $\gamma X$, then $g\left(x_{1}\right) \in T \subseteq E$ or $g\left(y_{1}\right) \in T \subseteq E$. Thus neither $x_{1}$ nor $y_{1}$ is in $\gamma X$. Therefore $x_{1}$ and $y_{1}$ are in $\operatorname{cl}_{\beta X}(\gamma X-X)-(\gamma X-X)$. This is a contradiction, since this set contains at most one point. Thus $\tau(T)=\beta T=\alpha T=E$.

(Only if) Suppose that $\tau: P^{*}(X) \rightarrow K(\delta X)$ is a lattice isomorphism and that for $x \neq y$ we have $\{x, y\} \subseteq K_{X}-\delta X$. Choose $p \in \gamma X-X$ and let $T(p, x)$ denote the quotient space of $\gamma X \cup\{x\}$ obtained by identifying $p$ and $x$. By 2.1(c) and 2.7, $T(p, x)$ has $P$ and evidently belongs to $P^{*}(X)$. The same is true of $T(p, y)$ and both of these spaces are distinct anti-atoms of the lattice $P^{*}(X)$; for if $T(p, x)=T(p, y)$, then $\beta T(p, x)=\beta T(p, y)$ by 3.2. Since $\beta T(p, x)$ is the compactification of $X$ obtained by identifying the points $p$ and $x$ of $\beta X$, and 
similarly for $\beta T(p, y)$, it follows from 3.8 (or more specifically from Lemma 1 of [9]) that $\beta T(p, x) \neq \beta T(p, y)$. Since $\tau$ takes anti-atoms to anti-atoms, it follows from Lemma 9 of [9] that there are points $a, b, c, d$ of $\beta(\delta X)-\delta X$ such that $\tau(T(p, x))=(a, b)$ and $\tau(T(p, y))=(c, d)$. [(a,b) is the compactification of $\delta X$ obtained by identifying the points $a$ and $b$ of $\beta(\delta X)$.] Now $T(p, x) \wedge T(p, y)$ is beneath precisely two anti-atoms of $P^{*}(X)$; yet if $a, b, c$ and $d$ are all distinct, then $(a, b) \wedge(c, d)$ is beneath six anti-atoms of $\underline{K}(\delta X)$. Hence not all can be distinct. Suppose $a=c$ and $a, b$ and $d$ are distinct. But then $(a, b) \wedge(a, d)$ is beneath three anti-atoms of $\underline{K}(\delta X)$. If also $b=d$, then $\tau$ is not one-to-one. In any case we have a contradiction.

4.4. Corollary. Let $X$ and $Y$ be l.p. and $\left|K_{X}-\gamma X\right|=\left|K_{Y}-\gamma Y\right| \leq 1$. If $\gamma X-X$ is homeomorphic to $\gamma Y-Y$, then $P^{*}(X)$ is isomorphic to $P^{*}(Y)$.

The authors have been unable to determine whether, for l.p. $X$ with $\left|K_{X}-\gamma X\right|$ $=1$, we have $P^{*}(X)=D^{*}(X)$. If this is true, (1) then this corollary and its converse are obtainable from Theorem 4.6 below. Otherwise the necessity of the full hypotheses of Theorem 4.5 may be called into question.

4.5. Theorem. Let $X$ and $Y$ be l.p. spaces. Assume that $D^{*}(X)=P^{*}(X)$ and $D^{*}(Y)=P^{*}(Y)$. If there is a homeomorphism $\sigma: K_{X} \rightarrow K_{Y}$ for which $\sigma[\gamma X-X]$ $=\gamma Y-Y$, then $P^{*}(X)$ is lattice-isomorphic to $P^{*}(Y)$.

Proof. By Magill's theorem, there is a lattice isomorphism $\tau: \underline{K}(\delta X) \rightarrow \underline{K}(\delta Y)$ such that for every $E$ in $\underline{K}(\delta X), \Im[\tau(E)]=\sigma[\Im(E)]$, where $\Im(E)=\left\{f^{\leftarrow}(p) \mid p\right.$ $\in E-\delta X$ and $f: \beta X \rightarrow E$ is the map fixing $\delta X$ pointwise $\}$.

Define $\hat{\tau}: P^{*}(X) \rightarrow P^{*}(Y)$ as follows: if $T \in P^{*}(X)$, then $\hat{\tau}(T)=\cap\{V \mid Y$ $\subseteq V \subseteq \tau(\beta T)$ and $V$ has $P\} \in P^{*}(Y)$. Claim. For each $T$ in $P^{*}(X), \beta(\hat{\tau}(T))$ $=\tau(\beta T)$. Let $f: \beta X \rightarrow \beta T$ fix $X$ pointwise. It suffices to show that $\mathcal{Y}[\beta(\hat{\tau}(T))]$ $=\mathcal{Y}[\tau(\beta T)]$, i.e. both compactifications of $Y$ yield the same decomposition of $\beta Y-Y$. By definition of $\hat{\tau}, Y \subseteq \hat{\tau}(T) \subseteq \tau(\beta T)=\alpha \hat{\tau}(T) \leq \beta \hat{\tau}(T)$. Thus

(A) $\mathcal{\Im}[\beta \hat{\tau}(T)]$ refines $\mathcal{Y}[\tau(\beta T)]$.

Let $h: \beta Y \rightarrow \beta(\hat{\tau}(T))$ and $g: \beta Y \rightarrow \tau(\beta T)$ be the maps fixing $Y$ pointwise and $k: \beta \hat{\tau}(T) \rightarrow \tau(\beta T)$ be the commuting map $k \circ h=g$. Note that $k$ fixes $\hat{\tau}(T)$ pointwise. But $\Im[\tau(\beta T)]=\left\{\sigma \circ f^{\leftarrow}(p) \mid p \in \beta T-T\right\} \cup\left\{\sigma \circ f^{\leftarrow}(p) \mid p \in T\right.$ $-X\}$. If $p \in \beta T-T$, then $\left|\sigma \circ f^{\leftarrow}(p)\right|=1$, and $p \in T-X$ if and only if $\sigma \circ f^{\leftarrow}(p) \cap \gamma X \neq \varnothing$.

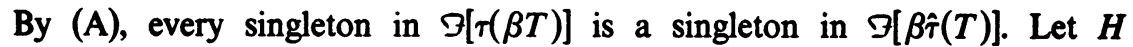
$\in \mathcal{Y}[\tau(\beta T)]$ with $|H|>1$. Then there is a $p$ in $T-X$ such that $H=\sigma \circ f^{\leftarrow}(p)$, so $H \cap \gamma Y \neq \varnothing$. Let $s \in H \cap \gamma Y$. Then $h(s)=t \in h[\gamma Y] \subseteq \hat{\tau}(T)$. Therefore $H=g^{\leftarrow}(t)=h^{\leftarrow} \circ k^{\leftarrow}(t)=h^{\leftarrow}(t) \in \mathcal{Y}[\beta \hat{\tau}(T)]$. Therefore $\mathcal{Y}[\beta \hat{\tau}(T)]$ $=\mathfrak{Y}[\boldsymbol{\tau}(\beta T)]$.

(1) Added in proof. Theorem: If $P=$ real compactness and $K_{X}-\gamma X$ is finite, then $D^{*}(X)$ $=P^{*}(X)$. 
It now follows that $\hat{\tau}$ is one-to-one. For $\hat{\tau}\left(T_{1}\right)=\hat{\tau}\left(T_{2}\right)$ if and only if $\beta \hat{\tau}\left(T_{1}\right)$ $=\beta \hat{\tau}\left(T_{2}\right)$, which is equivalent to $\tau\left(\beta T_{1}\right)=\tau\left(\beta T_{2}\right)$, thus equivalent to $\beta T_{1}=\beta T_{2}$ and hence equivalent to $T_{1}=T_{2}$. Moreover $\hat{\tau}\left(T_{1}\right) \leq \hat{\tau}\left(T_{2}\right)$ if and only if $\beta \hat{\tau}\left(T_{1}\right)$ $\leq \beta \hat{\tau}\left(T_{2}\right)$, which is equivalent to $\tau\left(\beta T_{1}\right) \leq \tau\left(\beta T_{2}\right)$, which in turn is equivalent to $\beta T_{1} \leq \beta T_{2}$, and finally equivalent to $T_{1} \leq T_{2}$.

Finally, $\hat{\tau}$ is onto. Let $V \in P^{*}(Y)$. Then $\beta V$ is in $\underline{K}(\delta Y)$, so there is a unique $\alpha X$ in $\underline{K}(\delta X)$ such that $\tau(\alpha X)=\beta V$. Let $T$ be in $P^{*}(X)$ and be contained in $\alpha X$. If $\alpha X=\beta T$, then $\tau(\beta T)=\beta \hat{\tau}(T)=\beta V$ and $\hat{\tau}(T)=V$. To establish this, we argue as before:

$$
\Im(\alpha X)=\left\{\sigma^{\leftarrow} \circ g^{\leftarrow}(t) \mid t \in V-T\right\} .
$$

Since $P^{*}=D^{*}, \sigma^{\leftarrow} \circ g^{\leftarrow}(t) \cap \gamma X \neq \varnothing$ is equivalent to $t \in V-Y$; hence these are precisely the sets that factor to points in $T$. The other sets, all singletons, go to $\alpha T-T$. Thus since every compactification of $T$ is a quotient of $\beta X, \alpha X$ is the largest possible compactification of $T$. Thus $\alpha X=\beta T$.

4.6. Theorem. If $X$ and $Y$ are l.p., then $P^{*}(X)$ is lattice-isomorphic to $P^{*}(Y)$ implies that $\gamma X-X$ is homeomorphic to $\gamma Y-Y$.

Proof. If $\gamma X-X$ is compact, the result follows from 4.3 and Magill's theorem. Otherwise as in Theorem 1 of [9], we construct a bijection between $K_{X}$ and $K_{Y}$ which carries those closed sets which hit $\gamma X-X$ onto closed sets which hit $\gamma Y-Y$ and vice versa. Since the bijection carries $\gamma X-X$ onto $\gamma Y-Y$, its restriction to $\gamma X-X$ is the desired homeomorphism.

4.7. Theorem. Let $X$ and $Y$ be l.p. spaces, and assume that $D^{*}(X)=P^{*}(X)$ and $D^{*}(Y)=P^{*}(Y)$. If $\gamma X-X$ and $\gamma Y-Y$ are $C^{*}$-embedded in $\gamma X$ and $\gamma Y$ respectively, then $\gamma X-X$ is homeomorphic to $\gamma Y-Y$ if and only if $P^{*}(X)$ is lattice-isomorphic to $P^{*}(Y)$.

Proof. "If" follows from 4.6. Conversely, since $\gamma X(\gamma Y)$ is $C^{*}$-embedded in $\gamma X \cup K_{X}\left(\gamma Y \cup K_{Y}\right)$, our assumptions imply that $\gamma X-X$ is $C^{*}$-embedded in $\operatorname{cl}_{\beta X}(\gamma X-X)=K_{X}$ (and similarly for $Y$ ). Thus $K_{X}$ is homeomorphic to $K_{Y}$ so the homeomorphism from $\gamma X-X$ onto $\gamma Y-Y$ extends to a homeomorphism of $K_{X}$ onto $K_{Y}$. Hence by $4.5, P^{*}(X)$ is lattice-isomorphic to $P^{*}(Y)$.

4.8. Corollary. Let $X$ and $Y$ be l.p. spaces. If either (a) $\gamma X-X$ and $\gamma Y-Y$ are compact, or (b) $\gamma X$ and $\gamma Y$ are normal and $D^{*}(X)=P^{*}(X)$ and $D^{*}(Y)=P^{*}(Y)$, then $\gamma X-X$ and $\gamma Y-Y$ are homeomorphic if and only if $P^{*}(X)$ and $P^{*}(Y)$ are lattice-isomorphic.

Proof. For (b), note that a closed subspace of a normal space is $C^{*}$-embedded [5].

5. Examples. If $P$ is taken to be compactness, the theory developed in the preceding sections essentially forms the content of Magill's paper [9]. We now consider three other topological properties satisfying the requirements for $\boldsymbol{P}$. 
(A) Realcompactness. It is well known that realcompactness satisfies 2.1; see Chapter 8 of [5] for example. Our first result is a positive one.

5.1. Theorem. Let $X$ and $Y$ be locally realcompact spaces and their Hewitt realcompactifications $v X$ and $v Y$ both be Lindelöf. Then $v X-X$ and $v Y-Y$ are homeomorphic if and only if $P^{*}(X)$ and $P^{*}(Y)$ are lattice isomorphic.

Proof. Each continuous image of $v X$ will be Lindelöf, and hence realcompact. Thus $P^{*}(X)=D^{*}(X)$, and similarly for $Y$. As every regular Lindelöf space is normal, the hypotheses of 4.8 are satisfied.

In this result, the assumption that the Hewitt realcompactifications were Lindelöf was used to insure $P^{*}=D^{*}$. Since realcompactness is not always preserved by perfect maps [3], [12], it seems reasonable that $D^{*} \neq P^{*}$ in general. To verify this, we have the following lemma and example.

5.2. Lemma. Let $X$ be any (completely regular Hausdorff) space and $Y$ be any Hausdorff space. Suppose for some compact subset $K$ of $\beta X$, there is a continuous surjection $h$ from $K$ to $Y$ such that $h^{\leftarrow} \circ h(x)=\{x\}$ for all $x$ in $K \cap X$. Then there is a completely regular Hausdorff space $T$ which contains $X$ densely, and a perfect map from $X \cup K$ onto $T$ which leaves $X$ fixed pointwise and satisfies $f^{\leftarrow} \circ f(p)$ $=h^{\leftarrow} \circ h(p)$ for all $p$ in $K$.

Proof. Let $T$ be the disjoint union of $X$ and $Y-h[K \cap X]$. Put $f(p)=p$ if $p \in X$ and $f(p)=h(p)$ if $p \in K-X$. The map $f$ is well defined since $h[K-X]$ $=Y-h[K \cap X]$. Next let $C$ be the set of all real-valued functions $l$ on $T$ such that $l \circ f$ is continuous on $X \cup K$, and let $T$ have the weak topology determined by $C$. By 3.8 of [5], $f$ is continuous on $T$. By 3.6 of [5], if $T$ is a $T_{1}$-space, then $T$ will be completely regular and Hausdorff. To show $T$ is $T_{1}$, it suffices to show that $f$ is a closed map.

Let $F$ be closed in $X \cup K$ and let $t \in T-f[F]$. Then $f^{\leftarrow}(t) \cap F=\varnothing$ and $f^{\leftarrow}(t)$ is compact. Thus $h[F \cap K]$ and $h\left[f^{\leftarrow}(t) \cap K\right]$ are disjoint compact sets of $Y$. Thus there exists $g$ in $C(Y)$ such that $\underline{0} \leq g \leq 1, g \circ h\left[f^{\leftarrow}(t) \cap K\right]=\{1\}$ and $g \circ h[F \cap K]=\{0\}$. Now $K \cup f^{\leftarrow}(t)$ is compact, so it is $C$-embedded in $X \cup K$. Thus there exists $k \in C(X \cup K)$ such that $\underline{0} \leq k \leq 1, k\left[f^{\leftarrow}(t)\right]=1$ and $k \mid K=g \circ h$. Now set $F_{1}=F \cap k^{\leftarrow}\left[\frac{1}{2}, \infty\right)$. Then $F_{1}$ is closed in $X \cup K$ and disjoint from $K$. Let $k_{1} \in C(X \cup K), \underline{0} \leq k_{1} \leq 1$, be such that $k_{1}\left[K \cup f^{\leftarrow}(t)\right]=\{1\}$ while $k_{1}\left[F_{1}\right]=\{0\}$. There exists $l \in C$ such that $l \circ f$ $=k k_{1}$. Then $l(t)=1$ and $l \circ f[F] \subsetneq\left(0, \frac{1}{2}\right)$. Thus $t \notin \mathrm{cl}_{T} f[F]$, so $f$ is a closed map. It is easy to verify that $f$ is perfect.

5.3. Example of a locally compact space $X$ for which $D^{*}(X) \neq P^{*}(X)$ when $P$ is realcompactness. By $8(\mathrm{I})$ of [5], there is a locally compact realcompact space $E$ (with nonmeasurable cardinal) and a continuous open map $g$ from $E$ onto $W$, the space of all countable ordinals. Let $g^{\beta}: \beta E \rightarrow W^{*}$ be the continuous extension of $g$. Set $X=W \times E$. Then $X$ is locally compact. Recall $[2,2.2]$ that if $Y$ is locally compact, realcompact and of nonmeasurable cardinal, and if $S$ is any space, then 
$v(S \times Y)=v S \times Y$. Hence $v X=W^{*} \times E$ (since $\left.v W=\beta W=W^{*}\right)$ and $v X$ $-X=\left\{\omega_{1}\right\} \times E$. If $f \in C(v X-X)$, define $g$ on $v X$ into the real numbers by $g(x)=f\left(\omega_{1}, x\right)$. Clearly $g \in C(v X)$ and so $v X-X$ is $C$-embedded in $v X$. Thus $K_{X}=\operatorname{cl}_{\beta X}(v X-X)=\beta(v X-X)=\left\{\omega_{1}\right\} \times \beta E$. Now let $h: K_{X} \rightarrow\left\{\omega_{1}\right\} \times W^{*}$ be the map defined by $h\left(\omega_{1}, g^{\beta}(p)\right)$. Since $K_{X} \cap X=\varnothing, h$ satisfies the hypotheses of 5.2. Let $T=X \cup\left[\left\{\omega_{1}\right\} \times W^{*}\right]$ and $f: X \cup K_{X} \rightarrow T$ be the space and map of that lemma. Now $X \cup K_{X}$ is realcompact, $f$ is perfect and $f \mid X$ is a homeomorphism. Hence by 3.7 of [10], $T$ is realcompact. If $T \in P(X)$, then it follows from 3.4 that $T \in P^{*}(X)$.

Since $h^{\leftarrow}\left[\left(\omega_{1}, \omega_{1}\right)\right]$ is disjoint from $v X$, the fact that $f^{\leftarrow} \circ f(p)=h^{\leftarrow} \circ h(p)$ for all $p$ in $K_{X}$ implies that $\left(\omega_{1}, \omega_{1}\right) \notin f[v X]$. Hence if $T \in P^{*}(X)$, then $T \in P^{*}(X)$ $-D^{*}(X)$ and we will be done.

Let $T_{1}$ be a realcompact subspace of $T$ that contains $X$. Since $f$ extends the inclusion map of $X$ onto $T_{1}$, we have $T_{1} \subseteq f[v X]$. Direct computation will show that $f[v X] \supseteq X \cup\left[\left\{\omega_{1}\right\} \times W\right]$, so that $T-\left\{\left(\omega_{1}, \omega_{1}\right)\right\} \subseteq T_{1}$. Thus $T_{1} \cap\left[\left\{\omega_{1}\right\}\right.$ $\times W]$ is a realcompact subspace of $\left\{\omega_{1}\right\} \times W^{*}$ which contains $\left\{\omega_{1}\right\} \times W$. Hence $T_{1}$ contains $\left\{\omega_{1}\right\} \times W^{*}=T-X$, whence $T_{1}=T$ and so $T \in P^{*}(X)-D^{*}(X)$.

(B) Almost realcompactness. Frolik [4] defines a Hausdorff space $X$ to be almost realcompact if given an ultrafilter $U$ of open subsets of $X$ such that $\cap\left\{\operatorname{cl}_{X} U: U\right.$ $\in \mathcal{U}\}=\varnothing$, there is a countable subfamily $\left(U_{n}\right)_{n \in N}$ of $U$ such that $\bigcap_{N} \operatorname{cl}_{X} U_{n}$ $=\varnothing$. Henceforth we shall take the term "almost realcompact" to mean "completely regular and almost realcompact". That almost realcompactness satisfies 2.1(a) and (b) is proved respectively in Theorems 5 and 7 of [4]. For 2.1(c), we appeal to 2.6 , and prove that

\subsection{Theorem. Almost realcompactness satisfies Mrowka's condition (W).}

Proof. We in fact prove the slightly stronger result that if $K$ is a compact subset of a space $X$ such that each closed subspace of $X$ disjoint from $K$ is almost realcompact, then $X$ is almost realcompact.

Let $\mathcal{U}$ be an ultrafilter of open subsets of $X$ such that $\cap\left\{\mathrm{cl}_{X} U: U \in \mathcal{U}\right\}=\varnothing$. By the definition of almost realcompactness, if suffices to find a countable subfamily $\left(U_{n}\right)_{n \in N}$ of $U$ such that $\cap_{N} \operatorname{cl}_{X} U_{n}=\varnothing$. Now $\cap\left\{K \cap \operatorname{cl}_{X} U: U \in U\right\}$ $=\varnothing$; hence since $K$ is compact, we can find $\left(U_{i}\right)_{i=1}^{n} \subseteq \mathcal{U}$ such that $\bigcap_{i=1}^{n} K \cap \operatorname{cl}_{X} U_{i}=\varnothing$. Put $U_{0}=\bigcap_{1}^{n} U_{i}$. Then $U_{0} \in U$ and $K \cap \operatorname{cl}_{X} U_{0}=\varnothing$. Whence by 3.11(a) of [5], there exists $g \in C(X)$ such that $g[K]=\{0\}$ and $g\left[\mathrm{cl}_{X} U_{0}\right]=\{1\}$. As almost realcompactness is closed-hereditary, our assumptions imply that $g^{\leftarrow}\left[\frac{1}{2}, \infty\right)$ is almost realcompact and $g^{\leftarrow}\left(\frac{3}{4}, \infty\right)=B$ is a cozero set in $g^{\leftarrow}\left(\frac{1}{2}, \infty\right)$. Since almost realcompactness is productive, and since every compact set is certainly almost realcompact, it follows from Proposition 1 of [7] that $B$ is almost realcompact.

Let $W=\{W \subseteq B: W$ is open and $W \supseteq U$ for some $U \in \mathcal{U}\}$. Notice that $U_{0} \in W$. We claim that $W$ is open ultrafilter on $B$ and $\cap\left\{\operatorname{cl}_{B} W: W \in W\right\}$ $=\varnothing$. Since $U$ is an open filter on $X$, we see that $W$ is an open filter on $\mathrm{B}$, and 
$\cap\left\{\mathrm{cl}_{B} W: W \in W\right\} \subseteq \cap\left\{\mathrm{cl}_{B} U: U \in U_{0}\right\} \subseteq \cap\left\{\mathrm{cl}_{X} U: U \in \mathcal{U}\right\}=\varnothing$. If $T$ $\subseteq B$ and $T$ is an open set not in $W$, then $T \cap U_{0} \notin U$, so as $U$ is an open ultrafilter, there is an $S \in U$ such that $T \cap S \cap U_{0}=\varnothing$. Now $S \cap U_{0} \in U$, so $S \cap U_{0} \in W$. Thus $W$ is an open ultrafilter on $B$ and our claim holds.

As $B$ is almost realcompact, there exist $\left(V_{n}\right)_{n \in N} \subseteq W$ with $\cap_{N} c_{B} V_{n}=\varnothing$. By definition of $W$, for each $V_{n}$ there is a $U_{n} \in U$ with $U_{n} \subseteq V_{n}$. Thus $\cap_{N} \mathrm{cl}_{B}\left(U_{0} \cap U_{n}\right)=\varnothing$. Since for every $n, \operatorname{cl}_{X}\left(U_{0} \cap V_{n}\right) \subseteq \mathrm{cl}_{X} U_{0} \subseteq g^{\leftarrow}[1] \subseteq B$, we see that $\operatorname{cl}_{X}\left(U_{0} \cap V_{n}\right)=c l_{B}\left(U_{0} \cap V_{n}\right)$. Thus for each $n, U_{0} \cap U_{n} \in U$ and $\cap_{N} \mathrm{cl}_{X}\left(U_{0} \cap U_{n}\right)=\varnothing$. Thus $X$ is almost realcompact.

Note that unlike compactness and realcompactness, almost realcompactness is not Mrowka's $E$-compactness for any space $E$; see [11].

(C) m-boundedness. Let $m$ be an infinite cardinal. In [6], a (completely regular Hausdorff) space $X$ is defined to be $m$-bounded if every subset $S$ of $X$ such that $|S| \leq m$ has compact closure. It is easy to show that $m$-boundedness satisfies 2.1. Since the continuous image of an $m$-bounded space is $m$-bounded, it follows that $D^{*}(X)=P^{*}(X)$ for any locally $m$-bounded space $X$. In Theorem 1.3 of [14], it is shown that the $m$-bounded reflection (denoted $m X$ ) of a space $X$ is the set $\left\{p \in \beta X\right.$ : there exists $A \subseteq X$ such that $|A| \leq m$ and $\left.p \in \operatorname{cl}_{\beta X} A\right\}$. As an immediate corollary to 4.8 , we have the following:

5.5. Theorem. Let $X$ and $Y$ be two locally $m$-bounded spaces such that either (a) $m X-X$ and $m Y-Y$ are compact, or (b) $m X$ and $m Y$ are normal. Then $m X-X$ and $m Y-Y$ are homeomorphic if and only if $P^{*}(X)$ and $P^{*}(Y)$ are latticeisomorphic.

5.6. Example of a space $X$ for which $m X-X$ is not $C^{*}$-embedded in $m X$. Let $m=\aleph_{0}$ and let $X$ be the discrete space of cardinality $\aleph_{1}$. Then $\aleph_{0} X-X$ is not $C^{*}$-embedded in $\aleph_{0} X$; see Theorem 2 of [13] for a proof.

\section{REFERENCES}

1. N. Boboc and Gh. Siretchi, Sur la compactification d'un espace topologique, Bull. Math. Soc. Sci. Math. Phys. R.P. Roumaine 5 (53) (1961), 155-165 (1964). MR 32 \#430.

2. W. W. Comfort, On the Hewitt realcompactification of a product space, Trans. Amer. Math. Soc. 131 (1968), 107-118. MR 36 \#5896.

3. Nancy Dykes, Mappings and realcompact spaces, Pacific J. Math. 31 (1969), 347-358. MR 41 \#7644.

4. Z. Frolik, A generalization of realcompact spaces, Czechoslovak Math. J. 13 (88) (1963), 127-138. MR 27 \#5224.

5. L. Gillman and M. Jerison, Rings of continuous functions, University Series in Higher Math., Van Nostrand, Princeton, N. J., 1960. MR 22 \#6994.

6. S. L. Gulden, W. M. Fleischman and J. H. Weston, Linearly ordered topological spaces, Proc. Amer. Math. Soc. 24 (1970), 197-203. MR 40 \#3511.

7. H. Herrlich and J. van der Slot, Properties which are closely related to compactness, Nederl. Akad. Wetensch. Proc. Ser. A 70 = Indag. Math. 29 (1967), 524-529. MR 36 \# 5898.

8. J. Mack, M. Rayburn and G. Woods, Local topological properties and one point extensions, Canad. J. Math. 24 (1972), 338-348. 
9. K. D. Magill, Jr., The lattice of compactifications of a locally compact space, Proc. London Math. Soc. (3) 18 (1968), 231-244. MR 37 \#4783.

10. S. Mrowka, Some comments on the author's example of a non-R-compact space, Bull. Acad. Polon. Sci. Ser. Sci. Math. Astronom. Phys. 18 (1970), 443-448. MR 42 \# 3749.

11. S. Mrowka and Z. Frolich, Perfect images of $R$ - and $N$-compact spaces, Bull. Acad. Polon. Sci. 19 (1971), 369-371.

12.—, On local topological properties, Bull. Acad. Polon. Sci. Cl. III 5 (1957), 951-956. MR 20 \#2678.

13. N. M. Warren, Properties of Stone-Čech compactifications of discrete spaces, Proc. Amer. Math. Soc. 33 (1972), 599-606.

14. R. G. Woods, Some $x_{0}$-bounded subsets of Stone-Čech compactifications, Israel J. Math. 9 (1971), 250-256. MR 43 \#3997.

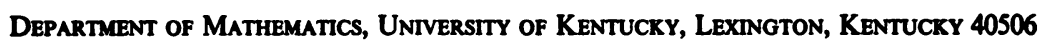

Department of Mathematics, University of Manttoba, Winnipeg, Manttoba, Canada 